\title{
Investigation of RF Signal Energy Harvesting
}

\section{Soudeh Heydari Nasab, Mohammad Asefi, Lutfi Albasha, and Naser Qaddoumi}

Department of Electrical Engineering, American University of Sharjah, P.O. Box 26666 Sharjah, UAE

Correspondence should be addressed to Lutfi Albasha, lalbasha@aus.edu

Received 3 May 2010; Accepted 25 October 2010

Academic Editor: Jianmin Miao

Copyright (C) 2010 Soudeh Heydari Nasab et al. This is an open access article distributed under the Creative Commons Attribution License, which permits unrestricted use, distribution, and reproduction in any medium, provided the original work is properly cited.

\begin{abstract}
The potential utilization of RF signals for DC power is experimentally investigated. The aim of the work is to investigate the levels of power that can be harvested from the air and processed to achieve levels of energy that are sufficient to charge up lowpower electronic circuits. The work presented shows field measurements from two selected regions: an urbanized hence signal congested area and a less populated one. An RF harvesting system has been specifically designed, built, and shown to successfully pick up enough energy to power up circuits. The work concludes that while RF harvesting was successful under certain conditions, however, it required the support of other energy harvesting techniques to replace a battery. Efficiency considerations have, hence, placed emphasis on comparing the developed harvester to other systems.
\end{abstract}

\section{Introduction}

$\mathrm{RF}$ harvesting is presenting itself as a viable source of energy. This is based on the notion that the level of transmitted and received signals and powers over the RF spectrum of frequencies has dramatically increased. The power that is required to charge contemporarily is mostly within microwatts or milliwatts [1]. This level of power can sometimes be generated using energy harvesters that can generate electricity using solar, mechanical vibration, temperature change, and body temperature energy [2]. Energy harvesters or scavengers are small systems that can generate electrical power using unlimited sources of energy $[1,2]$.

There are many signals of different frequencies in the surrounding that can be reclaimed. Even though these signals carry a small amount of energy, the possibility of using their rms power to charge or run a microchip can be studied. In this paper, utilization of air signals for energizing a low voltage circuit is investigated.

As the demand for remote and even disposable sensors increases, there is an increasing interest in battery-less systems that use energy harvesters $[3,4]$. Commercial prototypes are already on offer, for example, piezoelectric harvesters [5]. The main challenges in this field are the need for the harvesters to be of small size and also use of passive and low power components. In this work, the focus is on electromagnetic signal harvesting. A model for an air signal harvester is presented. The main components of the system are explained and the challenges faced are addressed. Finally, the future possibilities and potentials are discussed.

\section{Spectrum Harvesting}

The spectra of different areas have different characteristics that depend on many environmental variables. For instance humidity of the location and the distance from the signal transmitters will affect the results of spectrum measurements. Most users will generally fall into two main regions: an urbanized location such as a city or a less populated area such as countryside or desert.

To inspect the RF signal level of a low density spectrum, measurements were conducted using a spectrum analyzer (Rohde \& Schwarz FSP) and a dipole antenna. Figure 1 illustrates a snapshot of the measurements that were taken in an area close to the university campus.

The spectrum was measured for both indoor and outdoor locations (in random pattern). The overall results show that the highest power levels are within the GSM bandwidth $(920 \mathrm{MHz}$ to $950 \mathrm{MHz})$. The maximum power of signals for outdoor measurements was found to be 


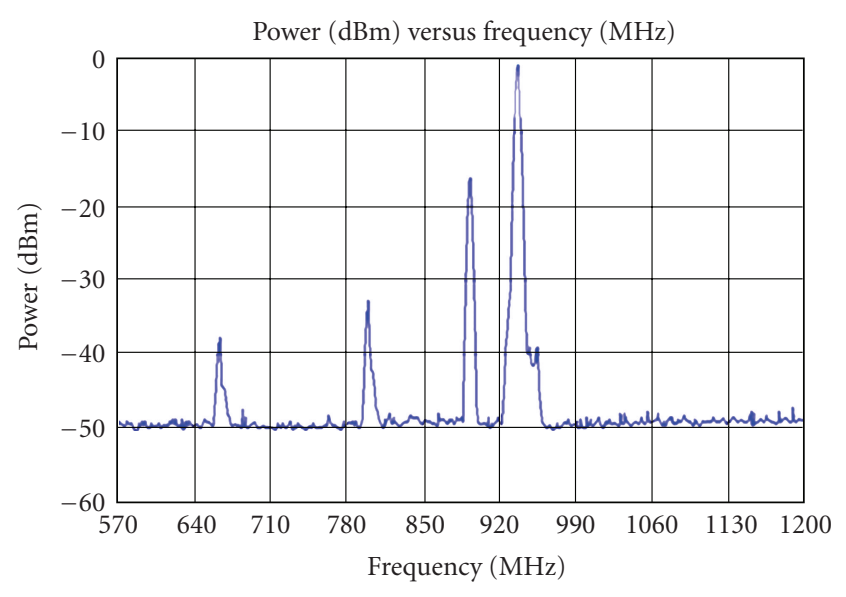

FIGURE 1: Snapshot of a low-density area Spectrum.

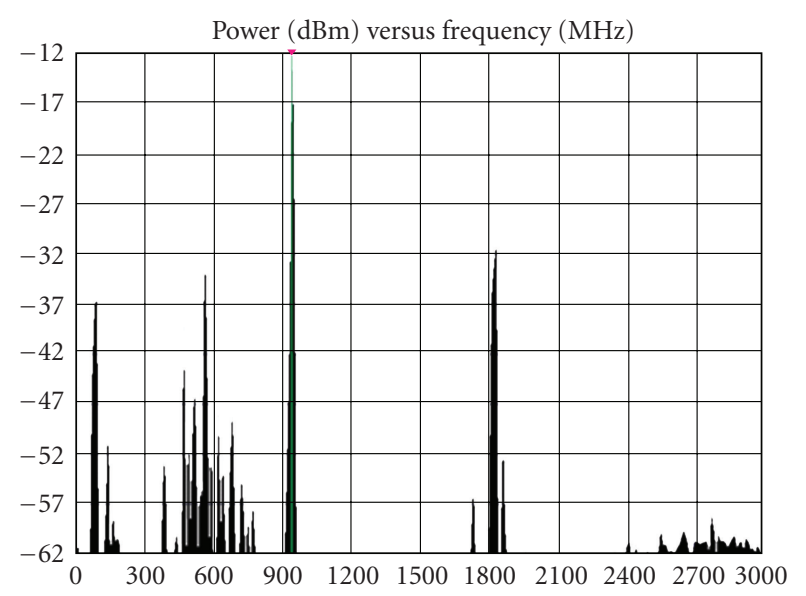

Figure 2: RF air signal Spectrum at a city location $(100 \mathrm{KHz}$ to $3 \mathrm{GHz})$.

approximately $-1 \mathrm{dBm}$. However, maximum signal level was about $-5 \mathrm{dBm}$ and even $-10 \mathrm{dBm}$ in some indoor locations. These results are illustrated, for the outdoor case, in Table 1. The measurements in this table are drawn from the results obtained from one location at different time instances.

As can be seen from the measurements in Table 1, the signal level at each frequency vary in a substantial manner at different instances.

Another spectrum analysis was run in a busy city location. Figures 2 and 3 illustrate the RF-air signals spectrums.

As can be observed the signals with maximum power level exist in the frequency range of $940 \mathrm{MHz}$ to $960 \mathrm{MHz}$.

In Table 2 the power variations of signals at different frequencies for different instances of time are also presented. The power levels in a busy city location are fairly constant.

From the spectrum of Figure 3 and the results shown in Table 2, it was found that the signals within $959 \mathrm{MHz}$ to $960 \mathrm{MHz}$ carry the maximum power with small variations within an acceptable range. Therefore, the harvester is to be designed with a GSM band antenna that has an approximate bandwidth of $40 \mathrm{MHz}$ and a center frequency of the harvester was selected to be $950 \mathrm{MHz}$.

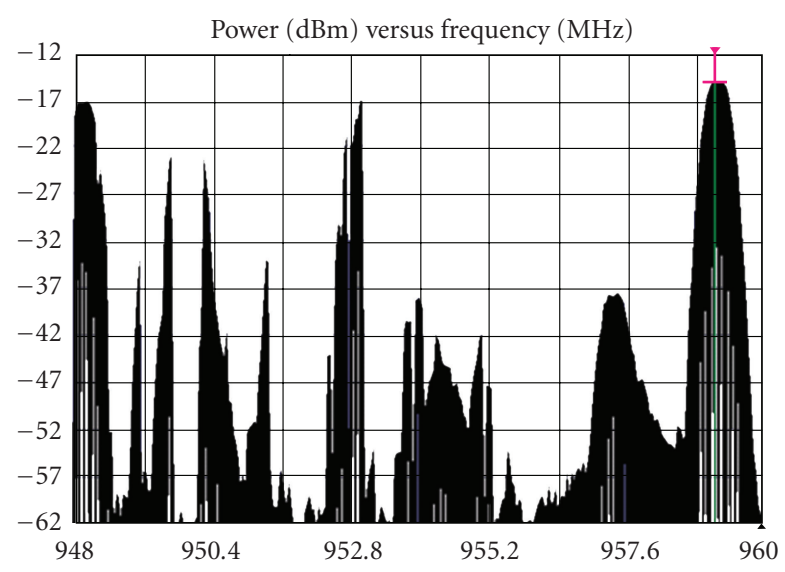

FIGURE 3: RF signal spectrum at a city location $(948-950 \mathrm{MHz})$.

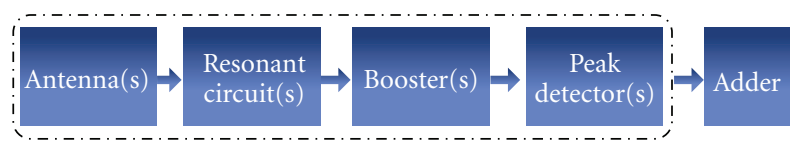

FIGURE 4: System diagram of RF harvester.

\section{Harvester Design}

The main target was the use of passive components in order to improve system efficiency. The size of the energy scavenger (harvester) in terms of number of targeted frequency ranges was another important point.

The input voltage to the harvester should be high enough to make the Schottky diodes forward biased. The input voltage to the harvester can be calculated by

$$
V=\sqrt{P \times Z}
$$

where $V$ : input voltage, $P$ : input power, $Z$ : line impedance ( $50 \Omega$ for this system).

The block diagram of the designed energy scavenger is shown in Figure 4.

A. Antenna(s) and Resonant Circuit(s). Since several signals of different frequencies are to be detected by this energy scavenger, there was a need for a double sided Microstrip GSM embedded antenna shown in Figure 5. This was followed by a few narrowband series resonant circuits used as RF selectors.

The air signals carry very low powers. In order to get more power from these signals, there was a need for a bigger antenna. The reason was that the directivity of the antenna increases with its size. The gain of the antenna is directly proportional to its directivity [6]

$$
G_{0}=e_{t} \cdot D_{0}
$$

where $e_{t}$ : the antenna efficiency (it depends on conduction, reflection and mismatch losses), $\mathrm{D}_{0}$ : antenna directivity.

Also, the increase in size of the antenna increases the antenna's bandwidth, the frequency range within which 
TABLE 1: Spectrum variations at different time instances.

\begin{tabular}{|c|c|c|c|c|c|c|c|c|}
\hline & \multicolumn{8}{|c|}{ Frequencies $(\mathrm{MHz})$} \\
\hline & 931.2 & 932.6 & 934 & 935.4 & 936.8 & 938.2 & 939.6 & 941 \\
\hline \multirow{9}{*}{ Power variations $(\mathrm{dBm})$} & -22.2 & -14.0 & -6.7 & -4.1 & -3.4 & -3.4 & -4.6 & -9.7 \\
\hline & -25.1 & -16.1 & -10.3 & -7.6 & -6.8 & -6.9 & -9.5 & -14.5 \\
\hline & -20.6 & -11.9 & -4.4 & -2.2 & -1.5 & -1.6 & -3.3 & -9.0 \\
\hline & -29.5 & -20.9 & -13.7 & -10.7 & -9.6 & -9.7 & -11.1 & -15.1 \\
\hline & -23.7 & -14.6 & -8.0 & -4.8 & -4.0 & -4.1 & -6.1 & -11.2 \\
\hline & -35.8 & -28.2 & -21.3 & -19.0 & -17.9 & -18.5 & -21.0 & -24.3 \\
\hline & -30.6 & -21.8 & -14.7 & -12.1 & -10.9 & -10.7 & -12.5 & -14.2 \\
\hline & -39.2 & -30.1 & -24.7 & -23.0 & -25.9 & -31.2 & -36.0 & -43.4 \\
\hline & -36.7 & -27.8 & -21.6 & -20.7 & -20.3 & -27.5 & -32.1 & -41.2 \\
\hline
\end{tabular}

TABLe 2: Frequency variations at different time instances.

\begin{tabular}{lccccccc}
\hline & & \multicolumn{3}{c}{ Frequencies (MHz) } \\
& 959.08 & 959.26 & 959.2 & 959.24 & 959.28 & 959.32 & 959.36 \\
\hline & -21.75 & -18.06 & -17.84 & -18.26 & -17.81 & -20.31 \\
Power variations $(\mathrm{dBm})$ & -17.91 & -14.29 & -14.07 & -14.38 & -13.97 & -16.10 & -26.02 \\
& -17.73 & -14.34 & -14.18 & -14.55 & -14.07 & -16.66 & -22.06 \\
& -18.34 & -15.15 & -14.92 & -15.18 & -14.80 & -16.94 & -22.44 \\
& -18.38 & -14.53 & -14.31 & -14.56 & -14.84 & -16.46 & -21.92 \\
& -18.17 & -14.78 & -14.59 & -14.88 & -14.38 & -16.87 & -25.76 \\
\hline
\end{tabular}

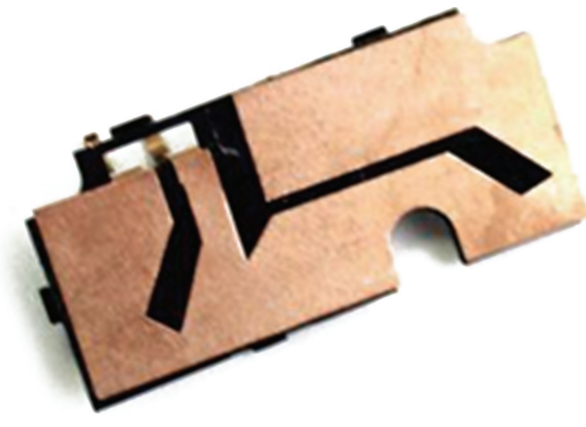

FIGURE 5: GSM embedded antenna.

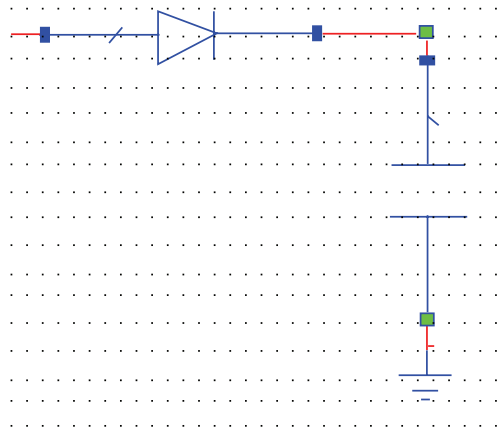

Figure 7: Passive peak detector.

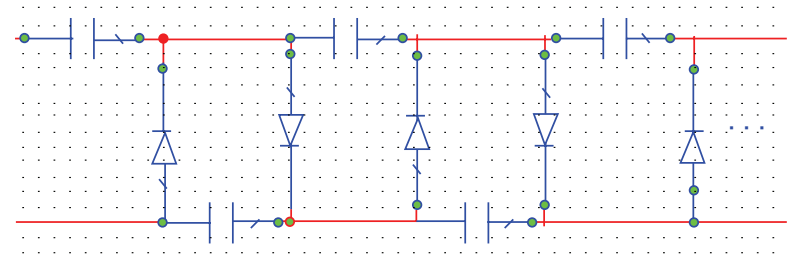

FIGURE 6: Villard voltage multiplier.

the antenna performance is efficient and it is usually centered at the resonant frequency [6]. Microstrip antennas have a bandwidth of $0 \%$ up to $5 \%$ of their center frequency.

Because of this, the power that can be gathered using this technique is going to be small. This fact limits the choice of the air signals to only one or two high power signals that carry the maximum possible detected energy. The signals to be detected can be Bluetooth signals (operating at $2.4 \mathrm{GHz}$ ) or GSM signals (operating at $960 \mathrm{MHz}$ ) since they appear to carry higher power than the other air signals and also they exist almost wherever there are cell phones. There will still be some other signals that fall within the bandwidth of the antenna. Since the signals detected by the antenna carry low power, a booster circuit was added to the system. A Villard voltage multiplier was used for this purpose.

B. Voltage Multiplier. Voltage multipliers are used to generate bias voltages of a few volts or tens of volts for purposes such as lightning safety testing. The most common type of voltage multiplier is the half-wave series multiplier, also called the Villard cascade (Figure 6) [7]. For this system, a Villard voltage multiplier is going to be used to boost up the received signal.

The output voltage that can be produced by an n-stage Villard cascade is $2 n$ and by selecting appropriate number of stages, any higher output voltages can be obtained. 


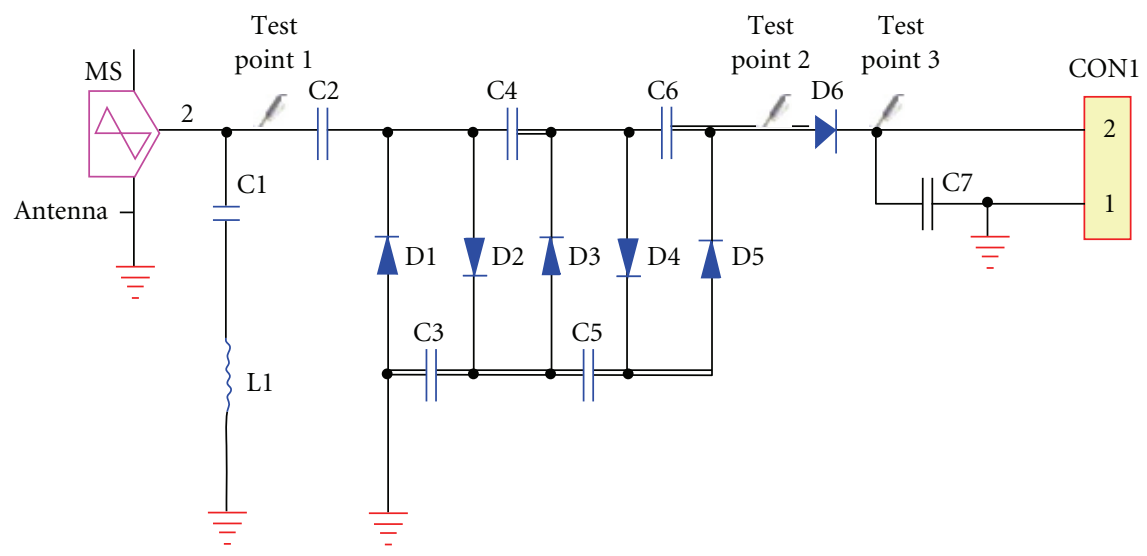

Figure 8: The RF harvester schematic.

However, if the output current increases, there will be a voltage drop across the capacitors due to alternating current which results in a lower input voltage for the succeeding stages. The formula for this voltage drop is given by [8]

$$
\Delta U=\frac{I}{f C}\left(\frac{2}{3} n^{3}+\frac{1}{4} n^{2}+\frac{1}{12} n\right),
$$

where $\Delta U$ : voltage drop, $I$ : output current, $f$ : input frequency, $C$ : capacitance, $n$ : number of stages.

As can be seen from (3), the voltage drop increases if the output current increases or input frequency and capacitance decrease. For the case where there is no control over the input frequency, the value of the capacitors should be selected properly. The ripples of the output of Villard multiplier can be found by [8]

$$
\Delta \delta=\frac{I}{f C} \cdot \frac{n(n+1)}{4} .
$$

The number of stages cannot be increased to any number. There are two main constraints. The first one is the decrease in the output current due to increase of the output voltage. The second issue is the restriction on the output voltage ripple. To find the most appropriate number of stages several simulations were run and it was found that for a six stage voltage multiplier the output ripples and the current are tolerable.

To find the appropriate value for the capacitors, several simulations were also run. Given the randomness and constant change of the input energy to the harvester, no discrete formula was found in the literature. In order to add signals of different frequencies, the output voltage of the Villard voltage multipliers should be converted into DC voltage using passive peak detectors.

Since a Villard multiplier only boosts alternating signals, it was decided to be placed prior to the DC converter.

C. Peak Detector. To convert the detected signals into DC, a passive peak detector was implemented. The simplest circuit consists of a Schottky diode with a low built in potential of approximately 0.2 Volts [9] and a capacitor as shown in Figure 7.
The overall circuit of the proposed harvester up to this stage is shown in Figure 8.

Higher output power can be gained by cascading several harvesters and adding their outputs together. To add these DC voltages, a passive adder is required.

\section{Simulations}

The capacitors of the harvester's multiplier are to be found. Since no empirical formula was found in literature, simulations were used. AWR Analog Office was used and the circuit in Figure 8 was tested with several different capacitors. Results are shown in the Figure 9.

As a result, $1 \mathrm{pF}$ capacitance was found to be the most appropriate. After the power of RF off-air signals was found for different frequencies and locations, the harvester's circuit was analyzed for different input powers. For this purpose, simulation signals of different powers were applied to the RF signal harvester at frequency of $1 \mathrm{GHz}$.

Figure 10 shows the results obtained for the power range of $-25 \mathrm{dBm}$ to $+6 \mathrm{dBm}$. The threshold level of the proposed harvester to $6 \mathrm{dBm}$. For harvesters with better sensitivity levels, techniques such as those used in RFID CMOS tags can be used [10].

As can be seen in Figures 10 and 11, with a small increase in the power of the input signal, the output voltage varies considerably.

The outputs shown in the graphs are for a single stage of this harvester. The voltage level is considerable for the high input powers, but it is not high enough. However, to further increase the obtained output voltage from the harvester without any further decrease in current, several stages of such circuit can be added together. To inspect the effect of addition, three of such harvesters were combined and a higher output DC voltage with higher current than that of a single stage with the same output was obtained. The result for this three stage harvester with three different input signals of $-20,-17$ and $-18 \mathrm{dBm}$ is shown in the graph of the Figure 12. 


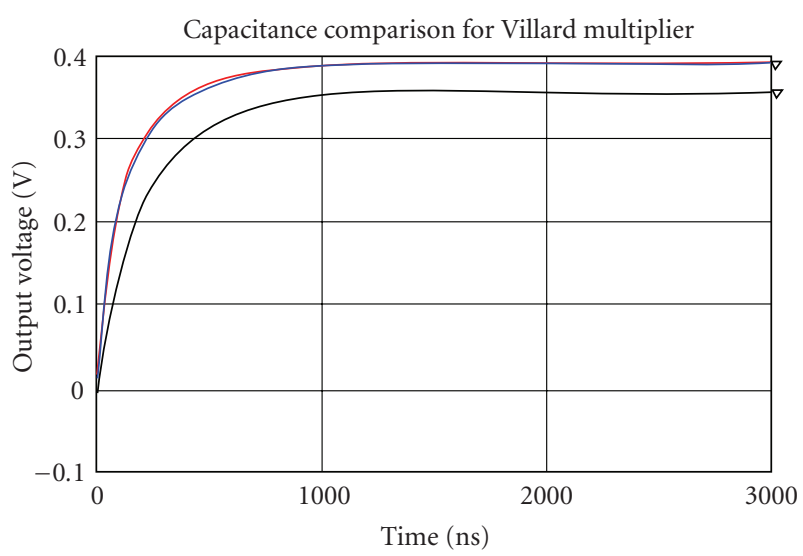

$\begin{array}{ll} & \text { Capacitance } \\ - & 1 \mathrm{pF} \\ - & 0.5 \mathrm{pF} \\ & 0.1 \mathrm{pF}\end{array}$

Figure 9: Different capacitances for Villard multiplier.

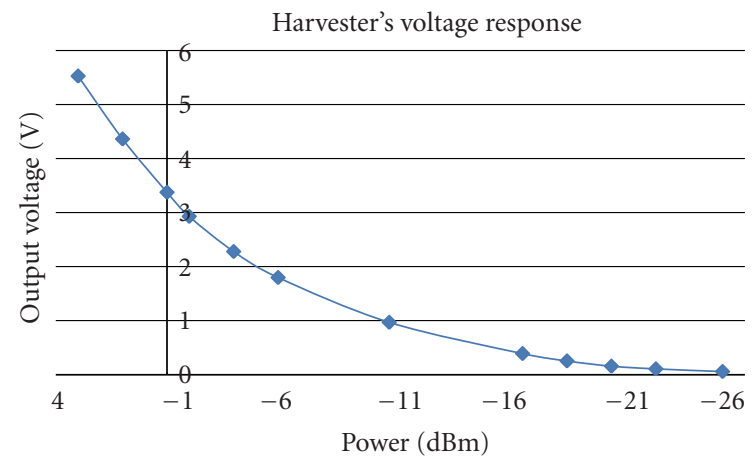

Figure 10: Harvester's voltage response to different input powers.

\section{Prototype Design}

To check the performance of the designed harvester a prototype was designed and implemented. This is shown in Figure 13.

The fabricated harvester was taken into different environments and its response to different input powers was analyzed. First, the harvester was left in an environment which was more than 500 meters away from the closest cellular phone base station. Also, there were not many mobile phones transmitting in the area were the tests took place. Figure 14 shows the voltage $(0.429 \mathrm{~V})$ obtained from these tests.

The harvester was successfully used to empower an LED. This is shown in Figure 15.

Furthermore, by using a nearby mobile phone as a transmitter, a calculator was successfully powered. A snapshot of this effect is shown in Figure 16.

As can be seen from the snapshot of Figure 16, there were two loads that are being powered with the proposed harvester at the same time in parallel; one is an LED and the other is a calculator. The battery and solar cells of the

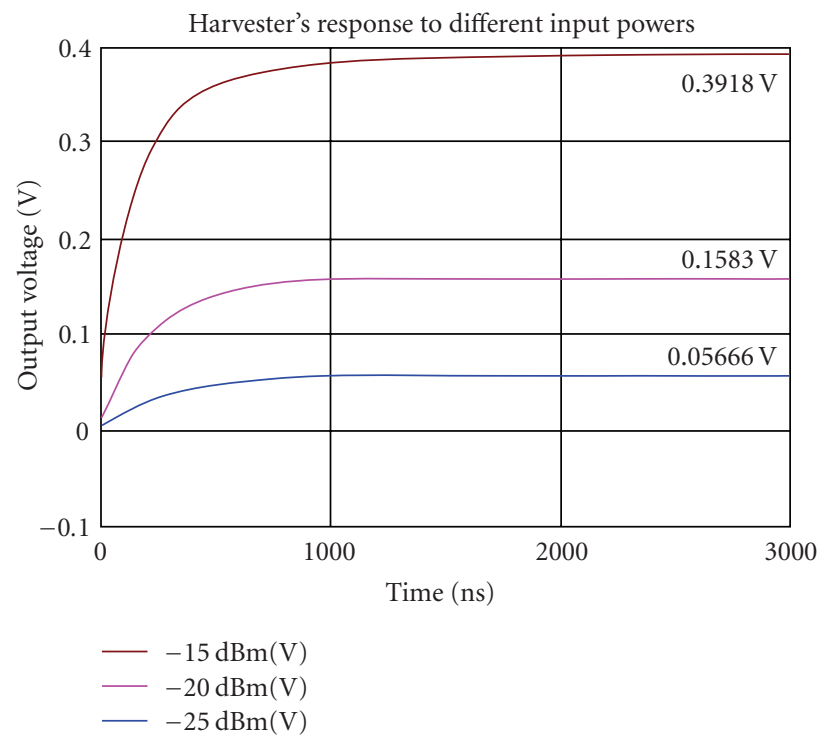

FIgURE 11: Harvester's Response to a $-15,-20,-25 \mathrm{dBm}$ Input.

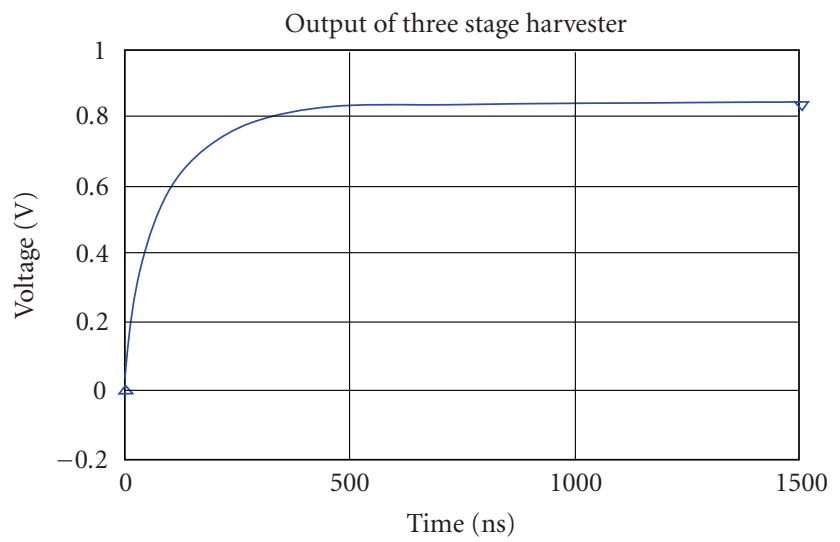

FIGURE 12: Three-stage harvester's output voltage.

calculator were removed. Generally speaking, efficiency from harvesters is not high. The efficiency of this circuit was found to be approximately $5 \%$ and for an input threshold of $-25 \mathrm{dBm}$ an output power of $158 \mathrm{nW}$ could be harvested. However, a comparison was conducted comparing this harvester with a piezoelectric harvester [11]. The results showed that while performance was comparable in terms of energy and efficiency, the cost of an RF harvester was considerably cheaper. This is shown in Table 3 .

A wireless mobile charger prototype was built and is currently being evaluated. The wireless charger comprised three different harvesters: a solar harvester, a piezoelectric harvester, and the proposed RF harvester.

\section{Conclusion and Future Work}

Based on measurements and simulations, it can be concluded that it is possible to use radiated, off-air RF signals as a source for energy harvesting. Even though the output powers of 
TABLE 3: Comparison between commercialized piezoelectric harvester and the proposed RF signal harvester.

\begin{tabular}{lcccc}
\hline Harvester & Size $(\mathrm{cm})$ & Output Voltage $(\mathrm{mV})$ & Cost $(\$)$ & Special considerations \\
\hline $\begin{array}{l}\text { Commercial Piezoelectric } \\
\begin{array}{l}\text { Prototype of proposed RF Signal } \\
\text { Harvester }\end{array}\end{array}$ & $4 \times 7.5$ & $0.25-0.3$ & 100 & Voltage based on tested measurements \\
\hline
\end{tabular}

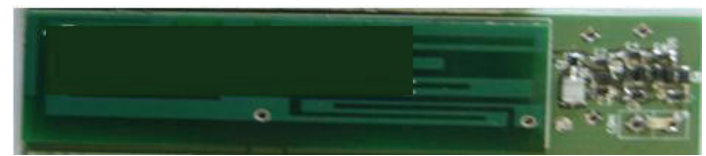

FIGURE 13: RF harvester's prototype.

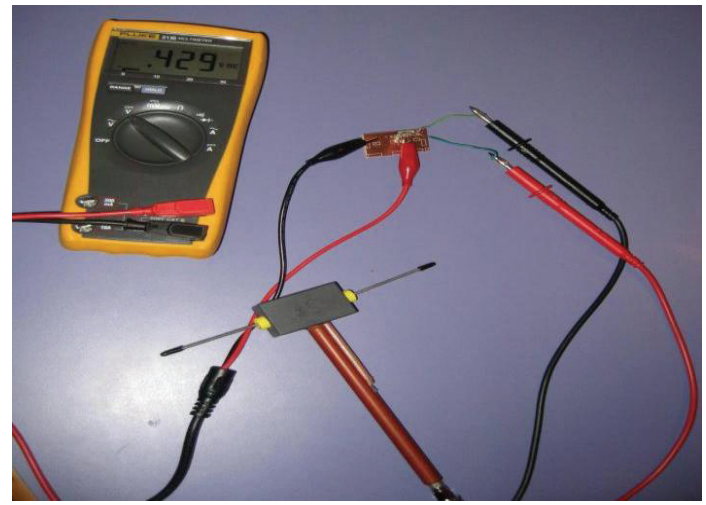

Figure 14: Voltage harvested by the RF signal harvester in an indoor area (more than 500 meters away from the base station).

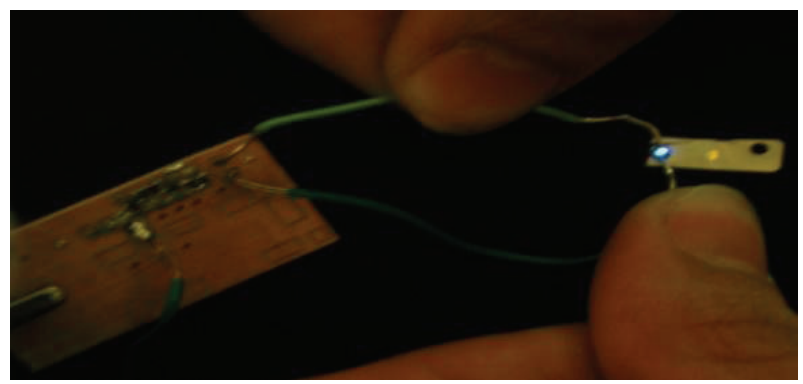

FIGURE 15: A LED, empowered by the proposed harvester.

such harvester are expectedly relatively low, it was sufficient for running low consumption sensors and switches.

Improvements on efficiency of the RF signal harvesting is important. This will enable more current to be re-cycled and operate low-power circuits. The possibility of using this harvester in energizing sensor networks appears to be the most practical use at the moment.

\section{Acknowledgment}

The authors would like to acknowledge and extend their gratitude to K.T.C. Company for their sponsorship.

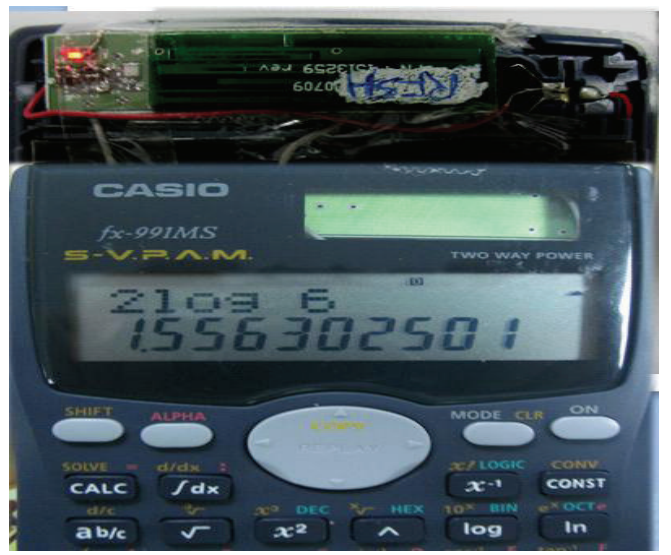

FIGURE 16: Back and front sides of the calculator running with the proposed RF signal harvester.

\section{References}

[1] M. Wright, "Harvesters gather energy from the ether, power lightweight systems," EDN, December 2006, http:// www.edn.com/article/CA6399099.html?industryid=2816.

[2] J. P. Thomas, M. A. Qidwai, and J. C. Kellogg, "Energy scavenging for small-scale unmanned systems," Journal of Power Sources, vol. 159, no. 2, pp. 1494-1509, 2006.

[3] N. Mokhoff, "Nano, MEMS are advancing energy harvesting technology," EE Times, June 2007, http://www.eetimes.com/ news/latest/showArticle.jhtml?articleID=199904312.

[4] Symbian News, "Nokai files patent for self charging mobile phone," March 2010, http://www.symbianfreak.com/news/ 010/03/nokia_files_patent_for_selfcharging_smartphone.htm.

[5] C. Hammerschmidt, "Race is on to develop energy harvest ICs," EE Times, October 2007, http://www.eetimes.eu/ 202400825.

[6] C. A. Balanis, Antenna Theory: Analysis and Design, John Wiley \& Sons, Hoboken, NJ, USA, 3rd edition, 2005.

[7] P. Scherz, Practical Electronics for Inventors, McGraw-Hill, New, NY, USA, 2nd edition, 2006.

[8] D. Kind and K. Feser, High-Voltage Test Techniques, Newnes, London, UK, 2nd edition, 2001.

[9] D. B. Rutledge, The Electronics of Radio, Cambridge University Press, Cambridge, UK, 1999.

[10] H. Nakamoto, D. Yamazaki, T. Yamamoto et al., "A passive UHF RF identification CMOS tag IC using ferroelectric RAM in $0.35-\mu \mathrm{m}$ technology," IEEE Journal of Solid-State Circuits, vol. 42, no. 1, pp. 101-109, 2007.

[11] S. Roundy and P. K. Wright, "A piezoelectric vibration based generator for wireless electronics," Smart Materials and Structures, vol. 13, no. 5, pp. 1131-1142, 2004. 

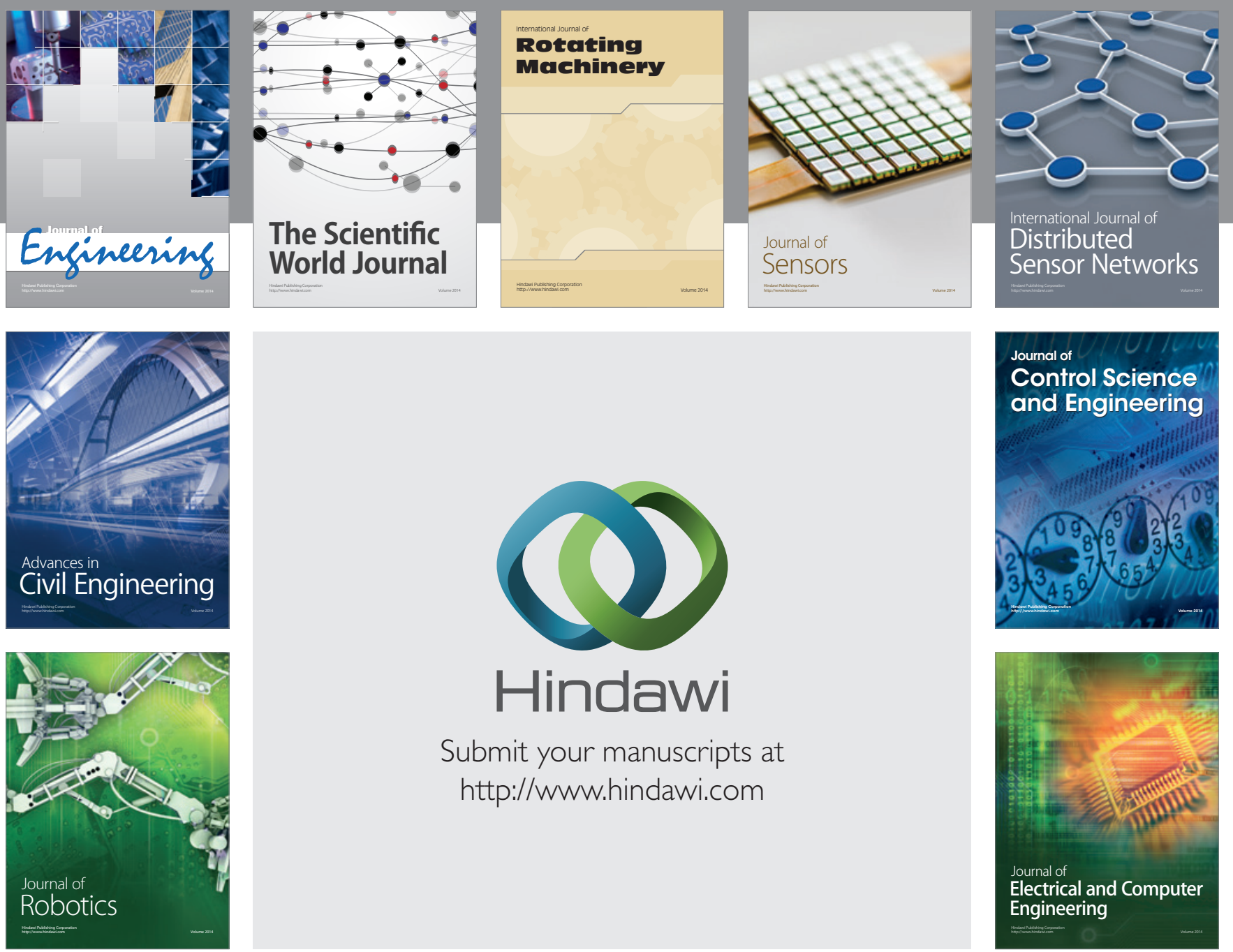

Submit your manuscripts at

http://www.hindawi.com
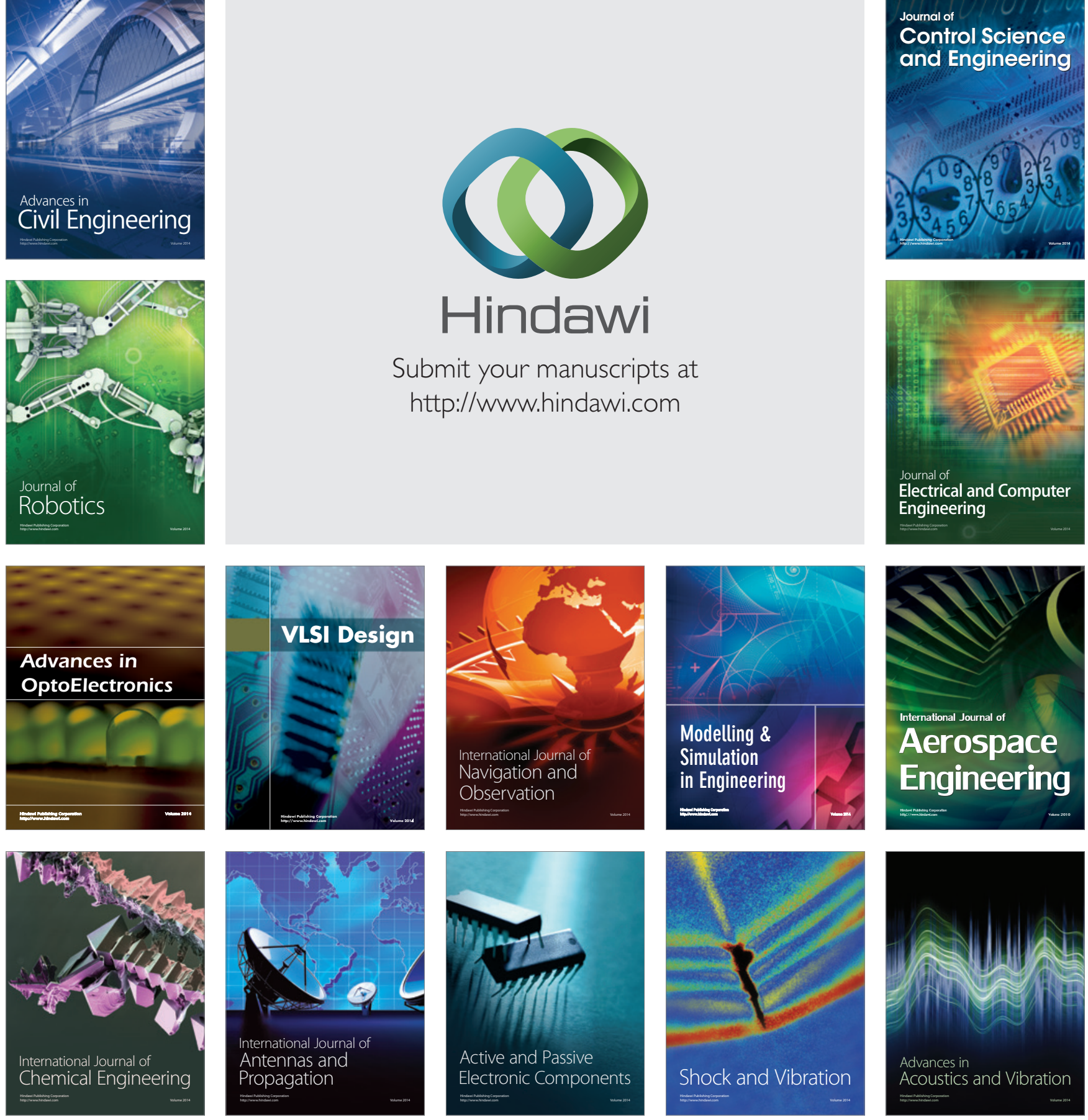